\title{
Anesthetic management for the surgery using tourniquet in aged people
}

\author{
Sungsik Park \\ Department of Anesthesiology and Pain Medicine, School of Medicine, Kyungpook National University, Daegu, Korea
}

Aging is a gradual process that results in functional maturation and a subsequent decline of in physiological function. Due to recent medical advances and to socioeconomic improvements in Korea, life expectancy has increased. In addition, declining fertility has increased the average age of the Korean population. According to government statistics [1], Korea entered the stage of an 'aging society' at year 2000, with $7 \%$ of total population aged 65 years and over; Korea will be reached the level of being an 'aged society' at year 2018, which means people over 65 years old will be over $15 \%$ of total population. Anesthesiologists have had increasing opportunities to treat older patients in the operating room, so need to be prepared to accommodate older patients.

In an article published in this issue of Korean Journal of Anesthesiology (KJA), Song et al. [2] present the effect of tourniquet deflation on hemodynamics and regional cerebral oxygen saturation in older-age patients. In this issue, the authors show conclusively that tourniquet deflation caused significant changes in hemodynamic and metabolic status, but did not change regional cerebral oxygen saturation; in addition, the authors recommended to monitor neurologic status as well as hemodynamic and metabolic status to avoid serious complications especially in aged patients.

In actuality, the effect of pneumatic tourniquet during lower extremity surgery has been investigated in at several institutions. The results of this article are not much different from previous studies. Choi et al. [3] described increased HR after deflation, but there was no change in HR in elderly patients under general anesthesia. Kim et al. [4] reported there were no changes in HR in elderly hypertensive patients. This result might be related with decreased activity in the autonomic nerve system and in cardiac reserve [5].

Immediately after tourniquet deflation, mean arterial pressure (MAP) decreased with cardiac output (CO) due to a shift of blood back to the limb and post-ischemic hyperemia. These changes may cause decreased perfusion to brain. On the other hand, transient increases in arterial carbon dioxide pressure (PaCO2) after deflation may increase cerebral blood flow about 50\%[6]. Increases in cerebral blood volume may result in secondary brain injury in patients with increased intracranial pressure. Therefore, it has become practice to monitor the central nervous system during orthopedic surgery when a tourniquet is in use. Compared with other monitoring tools, near-infrared spectroscopy (NIRS) is relatively simple, noninvasive, and reliable. Even though Song et al. observed no meaningful changes in regional cerebral oxygen saturation, the increased PaCO2 and decreased MAP and CO are enough reasons to monitor the CNS.

As mentioned above, aging is not only means declining of physiological functions that result in a decreased reserves, but also sometimes is combined with underlying diseases such as hypertension, diabetes mellitus, and cardiopulmonary disease, etc. Pre-existing concomitant diseases related with higher morbidity and mortality [7]. Therefore there are greater morbidity, mortality and recovery times from anesthesia for elderly patients than those for younger patients [8]. In this aspect, more research needs to be performed to manage older patients during perioperative periods in anesthetic research field.

Corresponding author: Sungsik Park, M.D., Department of Anesthesiology and Pain Medicine, School of Medicine, Kyungpook National University, Samduk-2 ga, Joong-gu, Daegu 700-721, Korea. Tel: 82-53-420-5863, Fax: 82-53-426-2760, E-mail: sspark@knu.ac.kr

(c) This is an open-access article distributed under the terms of the Creative Commons Attribution Non-Commercial License (http:// creativecommons.org/licenses/by-nc/3.0/), which permits unrestricted non-commercial use, distribution, and reproduction in any medium, provided the original work is properly cited. 


\section{References}

1. Korean National Statistical Office. Population projections for Korea 2001-2050. based on the 2005 population census. Daejeon, Korea National Statistical Office. 2006, pp 43-5.

2. Song IK, Kim DY, Kim YJ. The effect of tourniquet deflation on hemodynamics and regional cerebral oxygen saturation in aged patients undergoing total knee replacement surgery. Korean J Anesthesiol 2012; 63: 425-30.

3. Choi Y, Yang HS, Park SE, Kim DY, Kim HK. Tourniquet induced hypertension and vital sign changes in elderly patients associated with tourniquet use under general or epidural anesthesia for total knee arthroplasty. Korean J Anesthesiol 1999; 36: 653-9.

4. Kim KS, Min HK, Youn HJ, Cheong MA, Jun JH. The hemodynamic effects of a tourniquet application during knee surgery in elderly patients with hypertension. Korean J Anesthesiol 2006; 51: 695-700.

5. Ebert TJ, Rooke GA. Alterations in circulatory function. In: Geriatric anesthesiology. 2nd ed. Edited by Silverstein JH, Rooke GA, Reves JG, Mcleskey CH: New York, Springer. 2010, pp 137-48.

6. Hirst RP, Slee T, Lam AM. Changes in cerebral blood flow velocity after release of intraoperative tourniquets in humans: A transcranial doppler study. Anesth Analg 1990; 71: 503-10.

7. Lee EH, Do KJ, Yang HS. Anesthesia for orthopedic surgery in patients older than 80 years. Korean J Anesthesiol 2007; 52: 537-42.

8. Tiret L, Desmonts JM, Hatton F, Vourch G. Complications associated with anaesthesia: a prospective survey in France. Can Anaesth Soc J 1986; 33; 336-44. 